\title{
Design and implementation of Android phones-based home security system
}

\author{
Yuan Yuan ${ }^{1, a}$, Hao Zhang ${ }^{1, b}$, Wei Shen ${ }^{1, c}$ \\ ${ }^{1}$ School of Mechanical Electronic \& Information Engineering, China University of Mining and \\ Technology (Beijing), Beijing,100083, China \\ ayuanyuan_kingback@163.com, bzhhcumtb@126.com, ${ }^{\mathrm{c}} 535567928 @ q q . c o m$
}

Keywords: Smart home; Android; security monitoring; CC2530 chip; ZigBee.

\begin{abstract}
Security monitoring is one of the important development direction of intelligent city in the future, and monitoring based on Android makes the application more convenient and intelligent. This paper proposes a design and implementation of the smart home system based on Android, which use the Android smart phone as home monitoring terminal, use the STM32 controller ARM with Cortex-M kernel as the core to build the hardware platform, and use ZigBee wireless sensor technology to build a home network. The hardware platform and mobile monitoring terminal communicate through the web server for data and instructions transmission. Experimental result shows that this system can collect real-time temperature, vibration and other environmental information of house, and can display the video image. When an exception occurs, this system can start the automatic alarm module, thus to avoid damage to property.
\end{abstract}

\section{Introduction}

The Android platform is an open mobile phone software and hardware platform which launched by Google in 2007. It is a operating system of Linux based on free and open source code, mainly used in mobile devices, such as intelligent mobile phone and tablet computer [1]. A series of software running on Android system arises and covers all aspects of daily life, making the Android system existing more than a mobile device platform. In addition, 3G technology makes the transmission speed of the mobile network has been greatly improved in recent years, breaking the limit of the speed of the real-time monitoring system, and meeting the needs of users to make quick decisions [2].

Home security systems are based on housing as a platform, and can be treated as a system which has the function of comprehensive information interaction home security monitoring alarm that composed of the monitoring nodes, the web server and mobile monitoring. Its main functions such sa rapid transmission of alarm information, the large capacity storage of alarm information, the intelligent feedback control and remote control are recognized by users [3].

\section{General design of system}

Android platform-based home security system is composed of a series of low-power sensor for indoor monitoring systems, web server running on the PC side and monitoring software installed on the mobile phone.

Indoor monitoring system uses the CC2530 low-power wireless transceiver chips and sensors to build indoor LANs, using ZigBee Protocol[4] for data transmission. Data collected by temperature sensors, vibration sensors and cameras is gather through ZigBee2007/PRO protocol in the gateway.

Web server management system developed on the platform of Windows, using MVC (Model-View-Controller) model to build the framework, and using JSP+Struts+MYSQL technology to develop, thus providing data sharing service for mobile phone terminal system. The server is the hub of the whole system, providing service such sa the data transmit, the information feedback, the data temporary storage and so on. In addition , web server management system also has the function of user management and data backup management. The main framework of the Android platform-based home security system is shown in figure 1 . 


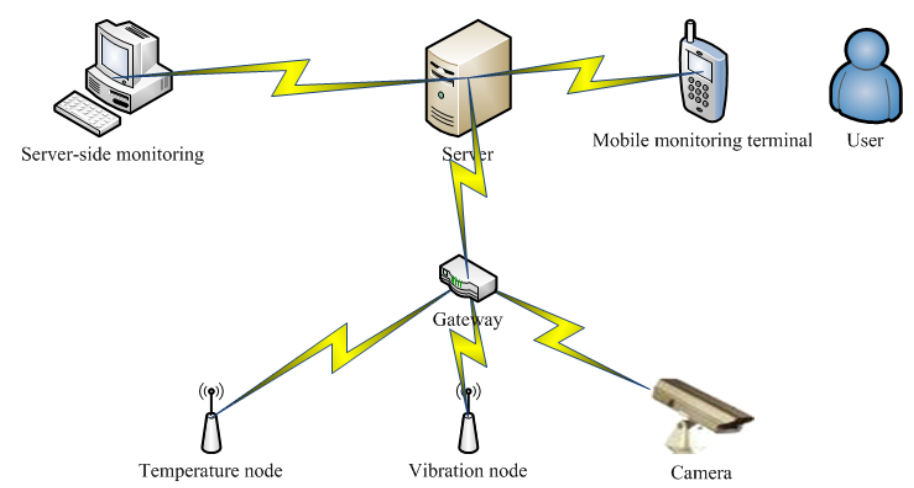

Fig.1: The overall framework of system

Mobile phone monitoring software developed on Android OS smartphone, using Eclipse for writing tests, calling parts of the Android API function library [5]. Mobile phone monitor is the part to interact directly with the users of the system, primarily used for user to check the real-time data of temperature and vibration nodes or the images of camera, after selecting the time period you can also view historical data for that time period.

Indoor monitoring system uses the SPI bus between the terminal and the web server for data exchange, web server and the mobile phone monitoring software through the use of the Web Service interface based on HTTP protocol implementation for data exchange [6].

\section{System implementation}

Indoor monitoring system implementation. Indoor monitoring systems chooses ZigBee network to form star-shaped mesh network. Nodes in the network can be divided into three roles of coordinator, router and terminal node. Coordinator is responsible for the creation and maintenance of LAN; router is responsible for data transfer between the coordinator and the terminal nodes; terminal node is responsible for collecting the data only. The embedded gateway is responsible for the establishment of ZigBee network [7], when network building is initialized, each sensor automatic search ZigBee network for data transmission. During the data transfer, the gateway from the heartbeat information to determine whether a terminal node or the network connection is working normally, heartbeat packet interval time is $5 \mathrm{~s}$. When the time interval is greater than $15 \mathrm{~s}$, the gateway can determine that the node is out of network.

The terminal node system using TI company's (System on Chip, SoC) CC2530 chip which combines the excellent performance of the leading RF transceiver, and supports a variety of serial communication protocols.

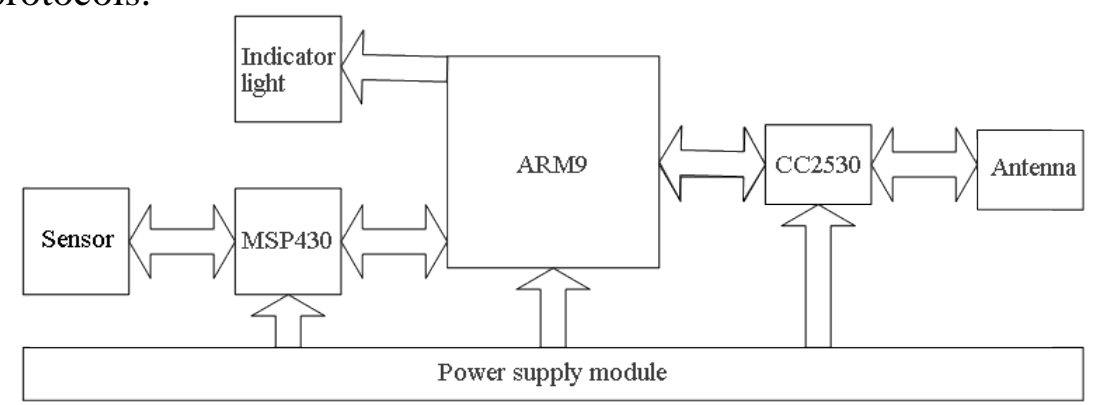

Fig.2: The terminal node hardware structure

Terminal node hardware mainly includes the power supply module, ARM9 chip, MSP430 series, temperature sensors, vibration node working status indicator light and CC2530 chip. Terminal node hardware structure as shown in figure 2, all the communication process is a two-way communication except the power supply module and status indicators. 
Cell phone monitoring program implementation. The monitoring terminal program is a mobile application that has real-time and historical data of view, real-time monitoring and other functions.

(i)Temperature and vibration data view

On the one hand, the system uses node-values to calculate the temperature or the vibration data at each point of the window to draw a data plan, and regions represented in different colors at different values, thus giving users a reflection of the whole situation of the window. On the other hand, line chart not only shows the trend of data for all nodes, but also notes the highest value and the data acquisition time of current data for users to check the situation.

(ii)Real-time image viewer

Real-time image viewer can show user the camera real-time picture of the room. In order to save bandwidth, and achieve faster transmission speed, during data transmission, the system will compress the picture, namely compress the BufferedImage objects which store image data, and then output byte array. When need to be displayed in mobile phone monitoring terminal, the mobile application will get the byte array from the server and save to ByteArrayInputStream, and then use the JPEGImageDecoder class to decode, thus finally decode the data back to the BufferedImage object. After seeing the image, if the user feels the picture Angle is not in the best position, he can click the "horizontal Angle Setting" or "vertical Angle setting" edit box to set the rotation Angle, and then click the "left", "right" or "up" or "down" button to order the camera rotation.

(iii)Alarm function

In order to ensure the safety warning role better, the system adopts three-level alarm mechanism, namely the gateway alarm, the server alarm and the mobile monitoring terminal alarm.

Mobile phone terminal will retain a certain number of data of monitoring nodes. Whenever a node has new data transmission over, the new data will be compared with historical data. If not found abnormal data, then display data normally. Otherwise, the system will trigger the alarm module.

The server-side implementation. The server using MVC framework for the realization of the web management system, which uses Web Service to communicate with the mobile phone monitoring terminal.

Server first detects deployment status while working. If user protection feature is enabled, then start a thread responsible for monitoring and receiving the indoor monitoring system to transmit the data. If a user is logged in mobile phone software, and call for data by the Web Service, server will searchs the data in database to meet the requirements of the user and returns the data to the mobile phone; when the mobile phone monitoring software user sends the control command to the hardware node, the server will transmits the control instruction and sends to the gateway. If the operation is successful, the success-result will be returned to the mobile phone monitoring software.

\section{The application and test}

In the lab, we carry out different tests. After opening the protection function, we can see the system running smoothly, even the most time consuming function of real-time image refresh delay is below 2s. Sensor could search to join the network and collect data for transmission after power on again.

In the process of testing, researchers pat near vibration sensors artificially to view the change of vibration data, and placed object whose temperature is below than the room temperature $10^{\circ} \mathrm{C}$ and higher than the room temperature $10^{\circ} \mathrm{C}$ near the temperature sensors to view the change of temperature data. The experimental results show that the abnormal data will trigger the alarm module. Mobile phone monitoring operation interface as shown in figure 3.

Figure 3.a shows the line chat operation interface of temperature, and figure 3.b shows the plan interface of temperature. Figure 3.c is a real-time camera image display interface. User can switch the room, the camera or change the period of time at any time by the buttons over the image to change the image. There are also a series of buttons below the image, which are convenient for the user to adjust the camera when in an emergency. The user can click on the "save" button to save the abnormal picture and click on the "alarm" button to call the pre-set alarm phone number. 


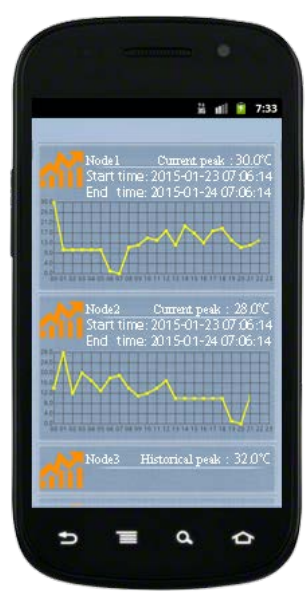

a. the line chart of temperature

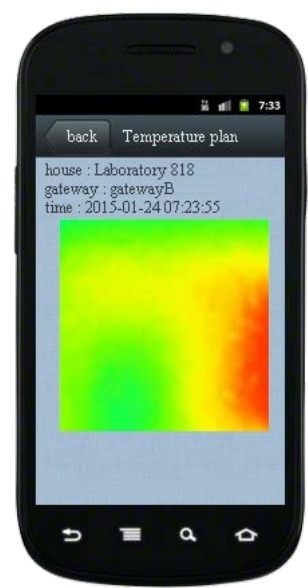

b. the plan of temperature

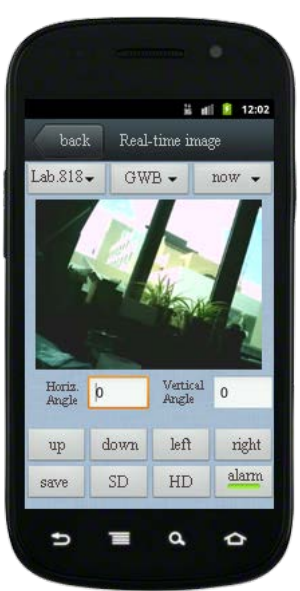

c. the real-time camera image

Fig.3: Mobile terminal operation interface

\section{Summary}

This paper designs and realizes a family security system platform which including indoor monitoring platform, web server and the android mobile phone monitoring. Simulation test and real machine test show that the system runs stably, and can monitor the real-time security situation in the house. This system not only has the ability to view real-time data but also the historical data can be recalled at any time for system and user to determine whether the warning is true; the unusual-photos saving and the one-button alarm functions can be implemented for quick alarm. The web server system uses the MVC design pattern so that the system will be maintained and expanded easily in the future. Accompanied by the moving transmission speed of the network and the increase of mobile processing performance, this system will have a broad application prospect.

\section{Acknowledgements}

This work was financially supported by National key foundation for exploring scientific instrument of China (No.2012YQ030126) and nuclear waste special scientific research project (No.FZ1402-08).

\section{References}

[1] ZHANG Shicheng. The research and development of Google application based on Android platform. Computer Knowledge and Technology, 2009, 5(28): 7959-7962.

[2] J.H. Wu, H. Xu and Y.H. Ding. Design and implementation of read-time monitor system in mobile network. Computer Engineering and Design, 2010, 31(10): 2196-2198, 2269.

[3] Y.Y. Li. Android platform based on intelligent home furnishing security system design. Manufacturing Automation, 2012, 34(6): 138-140.

[4] LI Wenzhong. Protocol stack ZigBee2007 / PRO experiment and practice. Beijing: Beihang University Press, 2009.

[5] Y.N. Zhang, L. Yang and L.M. Zheng. Remote video surveillance system based on Android mobile phone. Journal of Computer Applications, 2013, 33(z1): 283-286.

[6] K. Lan, Y. Zhang. Application of Android in remote medical information system. Journal of Computer Applications, 2013, 33(6): 1790-1792.

[7] DU Heng. Research on Android Smart Phone Home Security System. Video Engineering, 2013, 37(13): 161-165. 